\title{
Disappearing foot drop: a peroneal nerve ganglion
}

\author{
Paul Hartman ${ }^{1}$ and Douglas LeGay ${ }^{2}$ \\ ${ }^{1}$ Class of 2010, Faculty of Medicine, Dalhousie University \\ ${ }^{2}$ Department of Sport Medicine, Dalhousie University
}

\section{Abstract}

A 38 year old man presented with extreme left leg pain. He had been unsuccessfully treated for many months before his condition resolved on its own. After some time his pain returned in a more severe form with swelling on the lateral aspect of the knee. MRI showed ganglion cyst compressing the common peroneal nerve, which was successfully removed and most function has been restored. This case is unique in the disappearance and reemergence of his condition, which is novel to the literature

$\mathrm{C}$ ommon peroneal neuropathy, as result of cystic compression, is a rare condition with relatively limited research and documentation. Typically, this condition has presented with gradual development of weakness in the muscles of the lateral and anterior compartments of the lower leg which eventually resulted in foot drop. Generally speaking, the pathology of this condition is the result of compression, or invasion of the common peroneal nerve at the level of the fibular head.

In most reported cases, the diagnosis of this condition is delayed ${ }^{1-7}$. This has been attributed to the rarity of the condition, and usually takes place over time as other pathologies are ruled out. The normal differential diagnosis for a presentation of gradual onset weakness leading to foot drop includes radiculopathy involving the L5 nerve root, posttraumatic intraneural hemorrhage, and nerve sheath tumor.

Treatment for such the condition has favored microvascular removal of the cyst compressing the nerve ${ }^{1-7}$. With the removal of the cyst, function has reportedly returned slowly and most patients exhibit a gradual return to normal function $^{1-7}$. Some patients reported in the literature have regained only partial function, and one patient in the literature reviewed had the misfortune of having no return of function after cyst removal ${ }^{3}$.

This is a case study presented to further highlight the significance of this rare condition; it also adds a novel finding that is previously unreported in the literature.

\section{Case Report}

In April 2006, Mr. S., a 38 year old sailor presented with pain in his left knee extending into his lower leg, accompanied by sudden onset of foot drop. He was lacking sensation over the dorsum of the foot and up the anterior aspect of his lower leg; he also complained of parestheisa extending from his knee to the dorsum of his foot. No signs or history of trauma to the knee were noted, nor was there swelling at the knee.

Previous to this event, he had been suffering from pain in his lower leg that had been steadily increasing in intensity over a three month period. Pain was dull in the morning and intensity increased through the course of the day, exaggerated by stairs and ladders on the ship. The intensity had been severe enough to keep him awake at night, and had kept him from performing certain duties as part of his job. Ship medical officers, without evidence of trauma or other precipitating factors, told him to apply ice and take anti-inflammatories in order to control the pain.

The day prior to the onset of foot drop, he was working in a confined area, where he had been forced to crouch and kneel on his knees for the majority of the day, causing him discomfort in his knee throughout the day. He first recognized his foot drop upon waking the next morning. Mr. S. had an extensive history of back pain, resulting in occasional leave from work. Because of this history, compounded by difficulty in understanding and interpreting the condition he described, the ship medical officers sent him to have an MRI of his lumbar spine, believing that the condition may have resulted from pathology affecting the L5 nerve root. The MRI displayed a partially herniated disc affecting the L5 root, which was taken as the cause of Mr. S'. symptoms. With this diagnosis, he was referred to an orthopedic surgeon to determine if a surgical approach was necessary to correct his condition. The surgeon, not believing that his symptoms were a result of the herniated disk, sent him for an EMG study of the common peroneal nerve.

Meanwhile, Mr. S. began physiotherapy in an attempt to try to regain strength and mobility of the affected muscles. The physiotherapist involved in his treatment believed that his symptoms were being aggravated by an overly mobile fibular head that was irritating the nerve. In an attempt to lessen this condition, the physiotherapist began to wrap his knee in 
order to stabilize the fibular head.

In mid July 2006, Mr. S underwent EMG testing, which identified reduced conduction velocity at the common peroneal nerve, affecting the muscles in its distribution. At the time, this supported the belief that his condition was due to irritation of the common peroneal nerve by an overly mobile fibular head.

Intriguingly toward the end of July, with no firm diagnosis of his condition made, his foot drop resolved spontaneously, restoring most motor function. His pain had also subsided to a comfortable level. Sensation had yet to return, and he did still display some parestheisa over his anterolateral lower leg, extending down to his foot. This reversal of his condition was attributed to successful physiotherapy, no further investigation were performed, and he was deemed fit for work.

In September 2006, Mr. S returned with the same symptoms that he had presented with in the past. After a morning at work, he developed sudden onset of foot drop and pain. He now described his knee pain as excruciating and unbearable. Unlike the previous presentation, there was no relief from the pain through use of anti-inflammatories, and pain was constant throughout the day, leaving him sleepless at nights. In contrast to the first presentation, there was a distinct swelling seen on the lateral aspect of the knee.

He was told to attend physiotherapy for the same treatments that had worked in the past, but did not attend as the pain was too great and therapy caused too much discomfort. X-rays taken of his knee showed no joint effusion and a normal

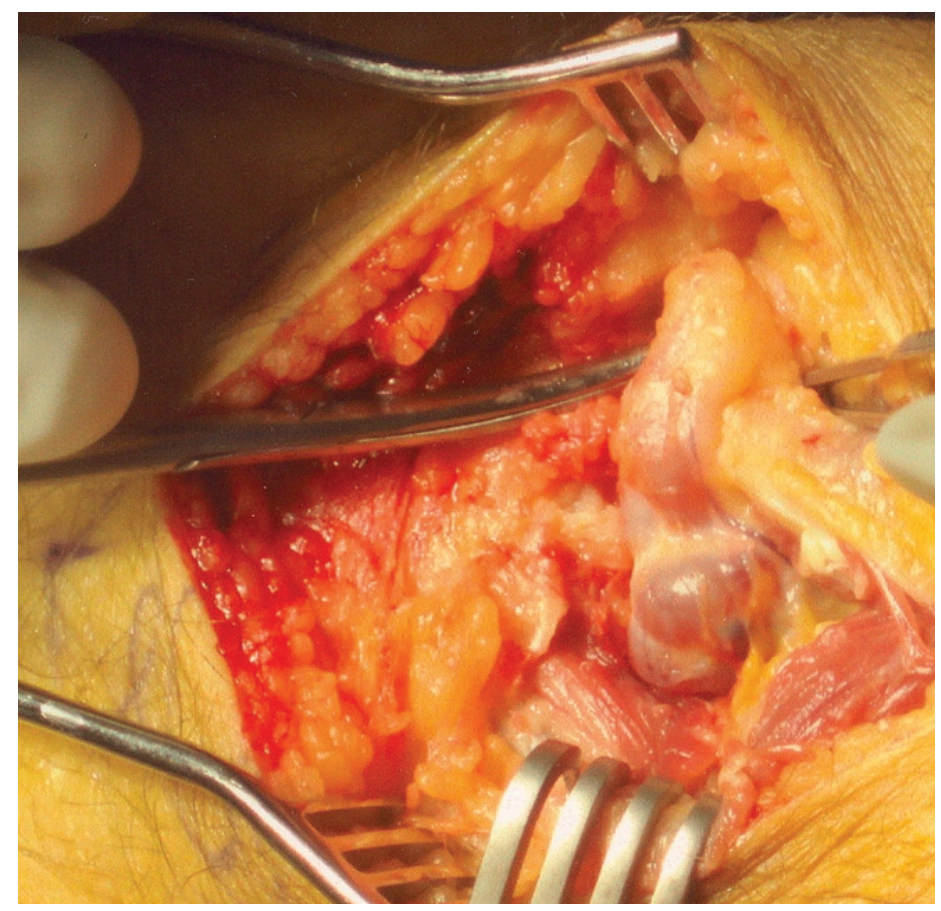

Figure 1. Intraoperative appearance of the intraneural ganglion causing neuropathy in the left common peroneal nerve tibiofibular joint, and he was thus referred for another EMG.

The second EMG, preformed in September 2006, showed a substantial decrease in function compared to his first EMG two months previously. The results showed denervation of the left peroneal motor nerves, with no such pathology associated with the left tibial nerve. Both the lateral and anterior muscle groups of the lower leg were affected, suggesting a lesion above the level of branching into deep and superficial peroneal nerves. In addition with no pathology associated with the tibial nerve, the lesion could be focused to the common peroneal nerve. This was further confirmed one month later by MRI, which defined a large mass appearing to compress the common peroneal nerve at the fibular head. All findings were consistent with the diagnosis of peroneal nerve ganglion. A surgical removal was suggested as the best option, and he was referred to another orthopedic surgeon.

At the initial consultation with the surgeon in November 2006, Mr. S. displayed grade 0 strength of the extensor hallucis longus (EHL), and demonstrated Tinel's sign of the peroneal nerve at the fibular head. At this stage the surgeon aspirated the cyst and removed $4 \mathrm{~mL}$ of gelatinous fluid. Aspiration did not relieve any of the symptoms.

Mr. S underwent surgery to remove the cyst in December 2006. At the time of surgery, it appeared to be an intraneural ganglion cyst. After excision, the cystic remains were analyzed histologically and it was confirmed to be a non malignant ganglion cyst demonstrating hyalinzed degeneration

Six weeks postoperatively, Mr S. was slowly regaining strength in the affected areas, and was assessed to have Grade $2 / 5$ power in the EHL, and $3+/ 5$ power in the anterior tibialis. He was able to heel walk. Sensation had not returned, and he reported that he continued to have parestheisa over the entire distribution of the common peroneal nerve.

At four months postoperatively he continued to improve; he was displaying grade $3 / 5$ in the EHL longus and $4 / 5$ power in the anterior tibialis. There still had been no improvement in sensation. He remains optimistic and is determined to regain full movement.

\section{Discussion}

There are many theories surrounding the development of cystic compression and invasion of the common peroneal nerve; most support development of a synovial cyst extending from the tibiofibular joint ${ }^{1-7}$. The cyst, growing over time, will eventually result in a compression neuropathy creating weakness in the extensor hallucis longus, extensor digitorum and anterior tibialis of the anterior compartment, as well as 
the peronei muscles of the lateral compartment ${ }^{1-7}$. Due to the slow progression and growth of the cyst from the tibiofibular joint, the onset of weakness is gradual and will eventually result in the development of foot drop ${ }^{6}$. This theory suggests that the development of the intraneural ganglion is a result of the cyst adhering to epineurium; following this, cystic material infiltrating the nerve causing damage to the nerve fascicles ${ }^{4,6,7}$.

Support for the above theory comes from the recognition of the small articular branch as the common starting point for synovial cyst infiltration ${ }^{2,5-7}$. It is believed that the synovial cysts will adhere and invade this branch and follow its course to the common peroneal nerve. In this case however, exploration of the articular branch showed no signs of cystic invasion, and it was therefore deemed unaffected and not the cause of the intraneural ganglion.

Other reports refer to a stalk that is shown to communicate between the cyst and the proximal tibiofibular joint ${ }^{1-7}$. Again, no stalk was seen to be directly communicating with the proximal tibiofibular joint. Thus, the pathology of the intraneural ganglion in this case must be a result of another mechanism.

Mechanisms for the development of intraneural ganglia at the peroneal nerve, outside of those proposed above, are not well explained. One theory suggests trauma causing intraneural hemorrhage, as a possible cause of intraneural ganglion ${ }^{5}$. Again, this theory is inconsistent with this case due to the gradual onset and lack of associated trauma to the affected knee. Gradual degeneration of the nerve sheath, leading to the development of the intraneural ganglion ${ }^{4-6}$, seems to be most consistent with the case presented here.

Review of literature failed to support a mechanism suggesting that irritation from the fibular head could induce intraneural hemorrhage. It has however been shown that, rarely, irritation by the fibular head can cause pain and motor loss ${ }^{8}$.

Timely diagnosis and treatment improves prognosis of this condition $^{7}$; we feel fortunate that, in light of our difficulties in diagnosing the condition, the outcome here was favorable.

\section{Conclusion}

This case was fascinating, and also troubling, due to the sudden disappearance, and later reemergence of the patient's foot drop. Knowing the pathologies proposed above, it seems unlikely that any of these truly fit the case as described above. To our knowledge, this is the first time such a finding has been described in the literature, adding to the delay in diagnosis. It is hoped that future cases may benefit from the case report presented here.

\section{REFERENCES}

1. Bakshi N, Chan KM, Wirganowicz PZ. Peroneal intraneural ganglion. Neurology 2005; 65: 1753

2. Coleman, Stuan. Et al. Intraneural Ganglion Cyst of the Peroneal Nerve Ac companied by Complete Foot Drop: A Case Report. The American Journal of Sports Medicine. 2001; 29: 238-241

3. Hersekli, Murat Ali. Et al. Synovial Cysts of Proximal Tibiofibular Joint Causing Peroneal Nerve Palsy: Report of Three Cases and Review of the Lit erature. Arch Orthop Trauma Surg. 2004; 124: 711-714

4. Pagnoux, Christian. Et al. Synovial cysts of the proximal tibiofibular joint: three case reports. Joint Bone Spine. 2002; 69: 331-333

5. Nucci, Francesco. Et al. Intraneural Synovial Cyst of the Peroneal Nerve: Report of Two Cases and Review of the Literature. Neurosurgery. 1990; 26(2): 339-344

6. Singh, Rajinder. Et al. Sudden Onset Foot Drop From Peroneal Ganglia. J. Orthopaedics. 2006; 3(4)

7. Rawal, Arvind. Et al. Compression Neuropathy of Common Peroneal Nerve Caused by an Extraneural Ganglion: A Report of Two Cases. Microsurgery. 2004; 24 (1): 63-66

8. Yilmaz, Erhan. Et al. Peroneal Nerve Palsy due to Rare Reasons: A Report of Three Cases. Acta Orthop Traumatol Turc. 2004; 38(1): 75-78

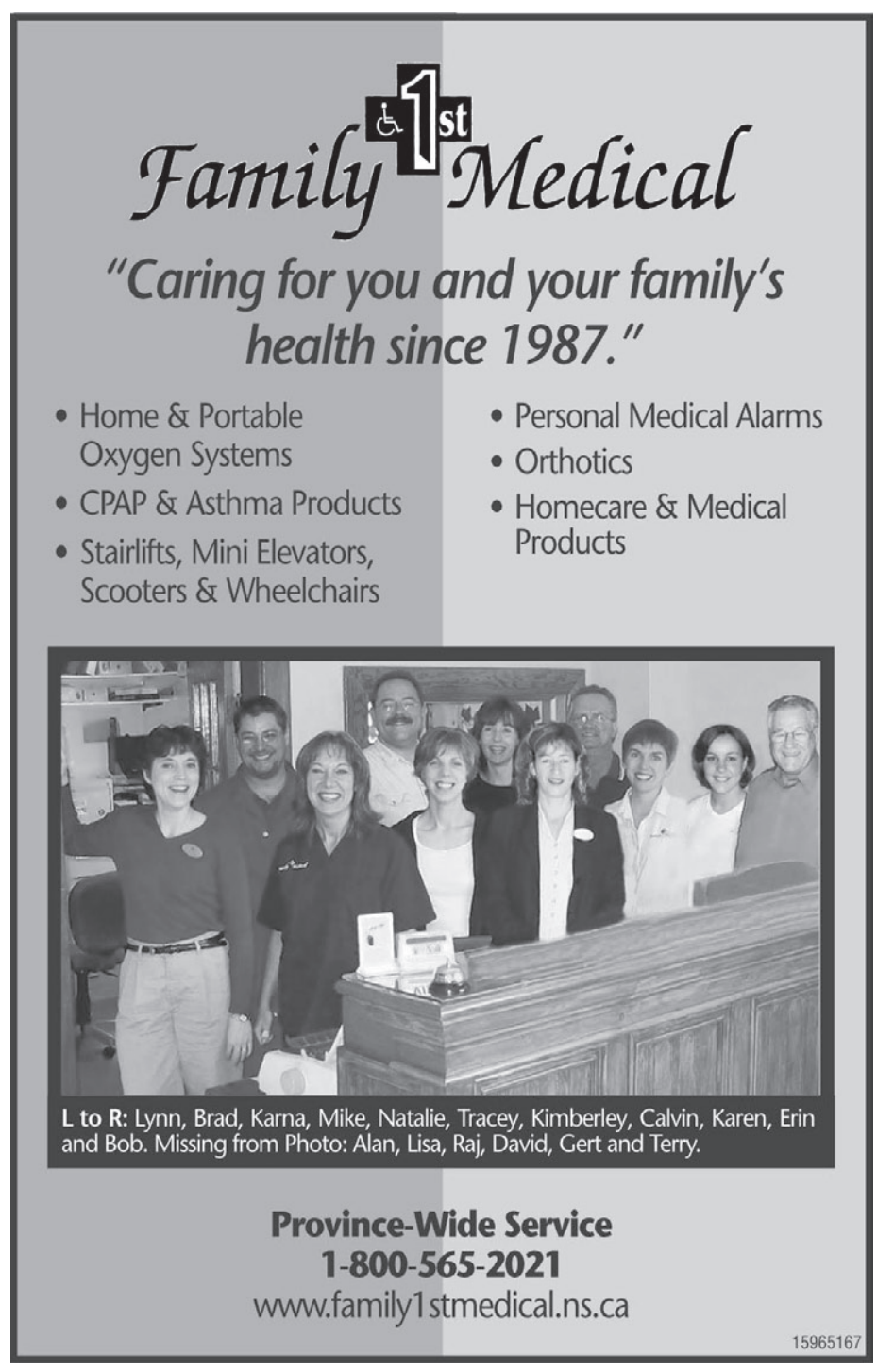

